\title{
Accurate Molecular Structure Determination by Femtosecond Degenerate Four-Wave Mixing
}

\author{
Dominique S. Kummli§, Hans M. Frey and Samuel Leutwyler \\ §SCS Poster Prize Winner
}

\begin{abstract}
Femtosecond degenerate four-wave mixing (fs-DFWM) is a Raman scattering type of rotational coherence spectroscopy that can be utilized for the measurement of highly accurate rotational and centrifugal constants of nonpolar molecules. The accuracy of the method has been tested using cyclopropane $\left(\mathrm{C}_{3} \mathrm{H}_{6}\right)$. The rotational and centrifugal distortion constants of this symmetric-top molecule are known to high accuracy. The fs-DFWM constants are currently only surpassed by those determined by dipole distortion microwave spectroscopy.
\end{abstract}

Keywords: High-resolution rotational constants · Molecular structure · Raman scattering ·

Rotational coherence spectroscopy

\section{Introduction}

The accurate determination of the geometrical structure of molecules is fundamental to the understanding of chemical bonding, electronic structure and reactivity [1]. The quantized rotational energy of a molecule depends inversely on its principal moments of inertia, which in turn depend on the molecular geometry [1-3]. The rotational level spacings of molecules can be measured in two complementary ways: (i) by high-resolution frequency-domain techniques such as rotational Raman spectroscopy [4], microwave spectroscopy [5], infrared spectroscopy or laser UV-VIS spectroscopy; (ii) by time-domain techniques based on rotational coherence spectroscopy (RCS) such as time-resolved fluorescence depletion and ionization depletion [6][7], pump-

\footnotetext{
${ }^{\star}$ Correspondence: Prof. Dr. S. Leutwyler Universität Bern

Departement für Chemie und Biochemie

Freiestrasse 3

$\mathrm{CH}-3012$ Bern

Tel.: +41316314479

Fax: +41316313994

E-Mail: samuel.leutwyler@iac.unibe.ch
}

probe photoionization, photoionization depletion, and time-resolved degenerate fourwave mixing (DFWM) [8-10]. The latter is a broadband coherent rotational Raman scattering technique. Unlike the other RCS techniques, DFWM does not depend on the existence of an excited electronic state, and in contrast to microwave spectroscopy, it does not presuppose the existence of a molecular dipole moment. It can be generally applied for the accurate determination of the rotational constants of the vibrational ground state of gas-phase molecules, with the exception of spherical tops, which lack pure rotational Raman transitions.

Increasing the molecular size and/or mass leads to increasing moments of inertia and decreasing rotational constants and spectral spacings. In the frequency domain, the rotational or rotational-vibrational transitions of large and/or heavy molecules may ultimately become too dense to be experimentally resolvable. An advantage of time-domain measurements is that increasing moments of inertia lead to increasing rotational periods; for an experimentally given time resolution this typically leads to increasing resolution. Here, we give a brief overview of the time-resolved DFWM technique, using cyclopropane as an example. Section 2 introduces the RCS theory: starting from the classical motion of a symmetric top, we proceed to a basic description of quantum beats and conclude with the fundamental expressions for degenerate four-wave mixing. Section 3 describes the experimental setup and Section 4 presents the results of our measurements on cyclopropane $\left(\mathrm{C}_{3} \mathrm{H}_{6}\right)[10]$.

\section{Elementary Picture of Rotational Coherence Spectroscopy [6][7]}

The classical motion of an oblate symmetric top is illustrated in Fig. 1. $\mathrm{I}_{||}$denotes the unique moment of inertia about the main symmetry axis (figure axis) of the molecule. For symmetric tops, the moment of inertia about any axis perpendicular to the figure axis is denoted $I_{\perp}$. The classical motion of the top is described by two motions, see Fig. 1: (i) the precession of the figure axis about the total angular momentum vector $\vec{J}$, at frequency $v_{1}=\omega_{1} / 2 \pi$; the Euler angle $\theta$ remains constant during the precession; (ii) the rotation of the molecule about its figure axis at angular frequency $\omega_{2}$. Fig. 1 shows a classical oblate top at times $\mathrm{t}=0$ and $\mathrm{t}=\pi / \omega_{1}$, half a precession period later. The frequencies $v_{1}$ and $v_{2}$ are given by [4]:

$$
\begin{aligned}
& v_{1}= \frac{|\vec{J}|}{2 \pi I_{\perp}}=\frac{\mathrm{J} \hbar}{2 \pi} \frac{4 \pi \mathrm{B}}{\hbar}=2 \mathrm{BJ} \\
& v_{2}= \frac{1}{2 \pi}|\overrightarrow{\mathrm{J}}| \cos \theta\left(\frac{1}{\mathrm{I}_{\| 1}}-\frac{1}{\mathrm{I}_{\perp}}\right)= \\
& \frac{\mathrm{K} \hbar}{2 \pi}\left(\frac{4 \pi \mathrm{B}}{\hbar}-\frac{4 \pi \mathrm{C}}{\hbar}\right)=2(\mathrm{~B}-\mathrm{C}) \mathrm{K}
\end{aligned}
$$


where $\mathrm{J}$ and $\mathrm{K}$ are the magnitudes of $\vec{J}$ and $\vec{K}$ in units of $\hbar$ and $\mathrm{B} \equiv \hbar / 4 \pi \mathrm{I}_{\| \mid}, \mathrm{C} \equiv \hbar / 4 \pi \mathrm{I}_{\perp}$ are the rotational constants corresponding to $\mathrm{I}_{\mathrm{II}}$ and $\mathrm{I}_{\perp}$.

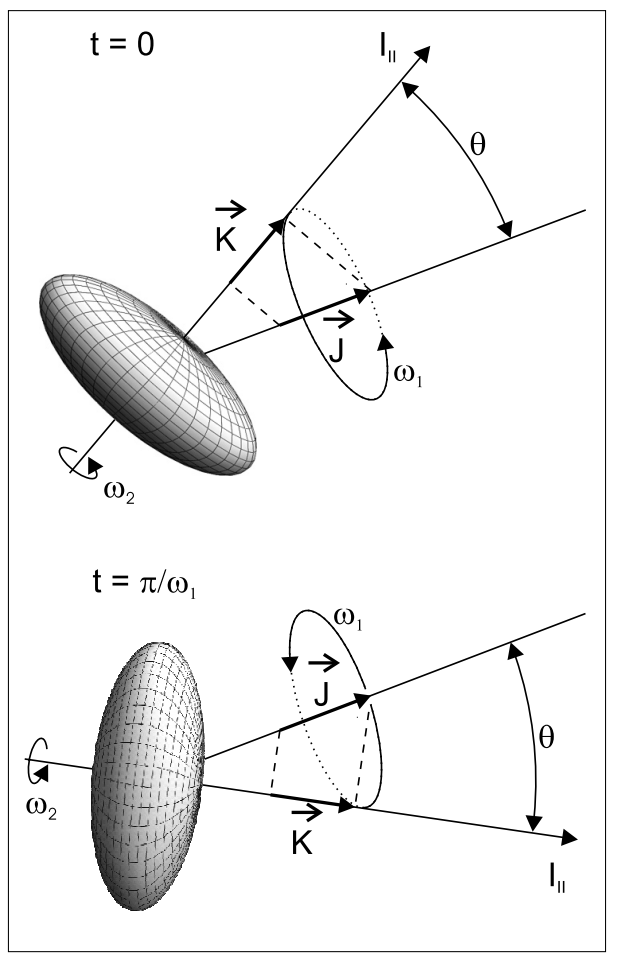

Fig. 1. Classical rotational motion of a rigid symmetric top. The top is shown at times $t=0$ and $t=\pi / \omega_{1} \cdot \vec{J}$ is the total angular momentum; its projection on the symmetry axis (figure axis) is called $\vec{K}$. The angular frequencies of precession and of rotation about the figure axis are $\omega_{1}$ and $\omega_{2}$, respectively.

A transition dipole parallel to the symmetry axis is only affected by the precession of the top. The radiation from this dipole is modulated by the precession frequency $v_{1}$ $=2 \mathrm{BJ}$ and its first harmonic. A transition dipole perpendicular to the symmetry axis is affected by the precession frequency $v_{1}$ and the rotation frequency $v_{2}$. For $v_{2}$ only the first harmonic $4(\mathrm{~B}-\mathrm{C}) \mathrm{K}$ is relevant, since a rotation about the symmetry axis of $\pi$ returns the dipole to its original direction. From this model one can derive the time and polarization dependences of the rotational coherence of molecules [11][12].

In rotational coherence spectroscopy [6][7][13] a polarized light pulse (the pump pulse) interacts with a dilute gas phase sample, preferentially with those molecules whose transition dipoles have large components along their polarization vector. At time-zero, this preferential interaction creates an initial orientational anisotropy of the molecular sample (Fig. 2b). As a result of the rotation of the molecules, the alignment dephases, but after characteristic time periods (typically $\sim 10$ to $100 \mathrm{ps}$ ) a periodic series of rephasings occur. The realignment is a manifestation of the fact that the molecules in the sample rotate at multiple integers of the fundamental frequencies obtained above.

RCS experiments are generally based on the following three schemes, which (i) involve polarized pump and probe processes; (ii) employ spectroscopic transitions (e.g. Raman transitions) with transition-dipole directions that are well defined in the molecule-fixed frame; (iii) monitor the observable as a function of time with a time resolution on the femto- to picosecond time scale. RCS is based on the quantum beat phenomenon, a time-domain manifestation of quantum interference. The time depen-

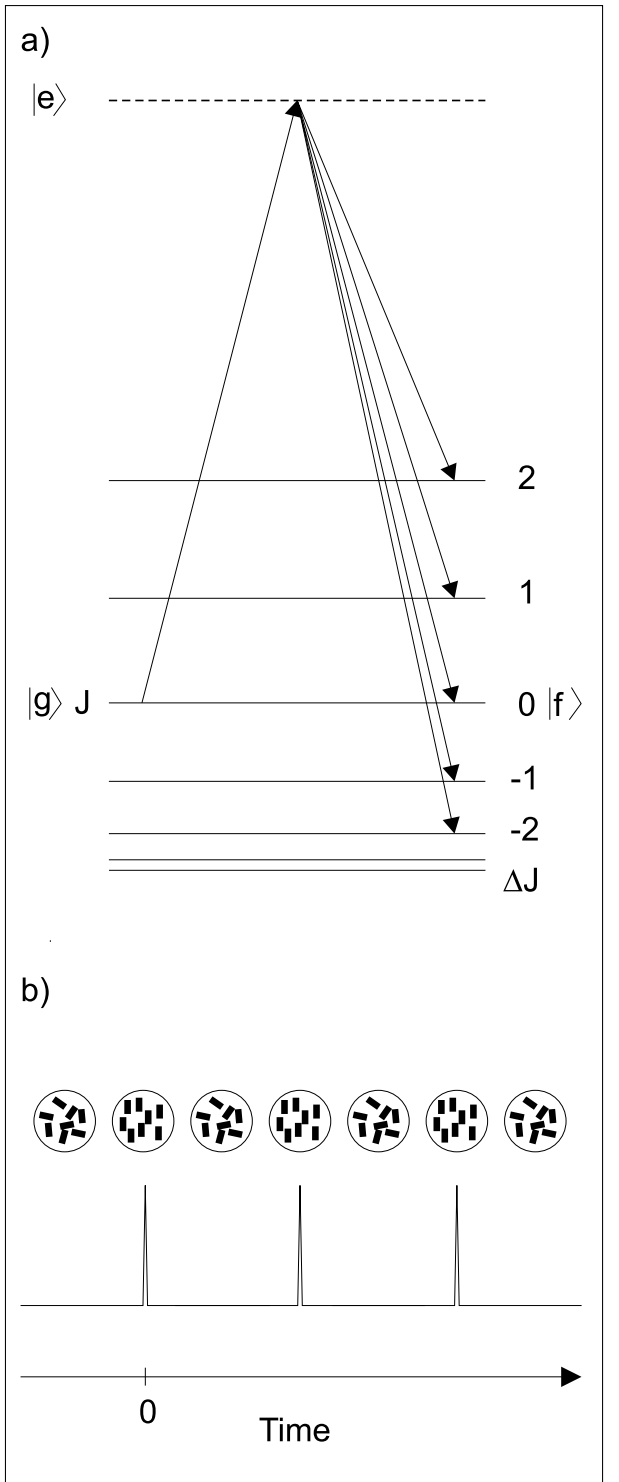

Fig. 2. a) Basic rotational level diagram for rotational Raman coherence spectroscopy. The ground state is denoted by $|\mathrm{g}\rangle$, and is characterized by the total angular momentum quantum number $J$. The short-lived (virtual) intermediate excited state $|e\rangle$ is denoted by a dashed line. The five final states are collectively denoted as If $\rangle$, together with the associated change of $J, \Delta J=2,1,0,-1,-2$. b) A schematic depiction of the time-dependent alignment of a gaseous sample of linear molecules and the associated RCS signal. dence, $\mathrm{I}(\mathrm{t})$, is determined by the sum of a large number of cosinusoidal modulation terms with time-independent coefficients $a_{i}$ :

$$
I(t)=\sum_{i} a_{i} \cos \left(2 \pi v_{i} t\right)
$$

If the $v_{\mathrm{i}}$ are uncorrelated, the cosine terms interfere destructively and $\mathrm{I}(\mathrm{t})=0$; however, if all the $v_{i}$ are integer multiples of a fundamental frequency $\Omega$ such that

$$
v_{i}=m_{i} \cdot \Omega
$$

then for all $i, \cos \left(2 \pi v_{1} t\right)=1$ whenever $t=$ $\mathrm{n} / \Omega$ (with $\mathrm{n}$ integer). At these times, all the cosine terms modulating $\mathrm{I}(\mathrm{t})$ interfere constructively, whereas at times significantly different from $n / \Omega$, destructive interference occurs. Thus, I(t) will be composed of equally spaced transients when Eqn. (4) applies.

In the time-domain Raman experiment shown in Fig. 2a, a molecule in a specific rotational state $|\mathrm{g}\rangle$ of the ground vibrational state is excited to a short-lived virtual (scattering) state le $>$ by the fs pump pulse. The fs dump pulse induces downward transitions to a set of final rotational states, collectively denoted as |f $\rangle$. For a symmetric top these can be labeled by the associated change of $J$, $\Delta J=2,1,0,-1,-2$. The rotational alignment induced by the space-fixed polarization of the fs pump and dump pulses is described by the distribution of quantum numbers $M_{J}$ spanned by the set of final states |f $\rangle$. The time evolution of the rotational wave packet is then monitored with the fs probe pulse, leading to the observable $\mathrm{I}(\mathrm{t})$. When using broad-band femtosecond laser sources (see below), the observable I(t) contains contributions from many initial rotational states.

In the femtosecond degenerate fourwave mixing (DFWM) scheme [6][810][14-17], three laser beams are focused into the Raman-active medium (Fig. 3a). The three fields are degenerate, i.e. identical in terms of pulse envelope and frequency components. The incident laser pulses $\mathrm{E}_{1}$, $E_{2}$ and $E_{3}$ interact in the so called 'forward box' [18][19] arrangement shown in Fig. 3a. This setup satisfies the phase-matching condition and also allows to spatially separate the incident pulses $\mathrm{E}_{1}, \mathrm{E}_{2}$ and $\mathrm{E}_{3}$ from the coherent Raman signal. In the crossing region of the pump and dump pulses $E_{1}$ and $\mathrm{E}_{2}$, their electric fields are spatially modulated due the mutual interference, see Fig. $3 \mathrm{~b}$. The molecules in the interaction region experience an electric field intensity that varies with their position, which leads to the formation of a transient alignment grating in space [20]. It corresponds to a superposition of the molecular response of all 
the rotational levels involved. The transient grating is then probed by Bragg scattering of the third laser pulse $\mathrm{E}_{3}$. The DFWM signal intensity, $\mathrm{I}_{\mathrm{DFWM}}$, resulting from the interaction between the three laser pulses is given by

$$
\mathrm{I}_{\text {DFWM }}(\tau)=\int_{-\infty}^{\infty}\left|\mathrm{P}^{(3)}\left(\overrightarrow{\mathrm{k}}_{\mathrm{s}}, \mathrm{t}\right)\right|^{2} \mathrm{dt}
$$

where $\mathrm{P}^{(3)}\left(\overrightarrow{\mathrm{k}}_{\mathrm{s}}, t\right)$ is the time-dependent thirdorder polarization. In case of the phase matching condition $\overrightarrow{\mathrm{k}}_{\mathrm{s}}=\overrightarrow{\mathrm{k}}_{\mathrm{a}}-\overrightarrow{\mathrm{k}}_{\mathrm{b}}+\overrightarrow{\mathrm{k}}_{\mathrm{c}}$ it can be written as

$$
P^{(3)}\left(\vec{k}_{s}, t\right)=E_{3}(t) \int_{-\infty}^{\infty} E_{1}(t) E_{2}(t) \sum_{n} b_{J} e^{i \omega_{J} t-\Gamma}
$$

where $b_{J}$ is an intensity factor that depends on the rotational level. It includes the populations given by the Boltzmann distribution, degeneracies and nuclear spin statistics, the orientation-integrated Raman transition probability (Clebsch-Gordan factors) and the laser bandwidth; $\omega_{\mathrm{J}}$ is the transition en-

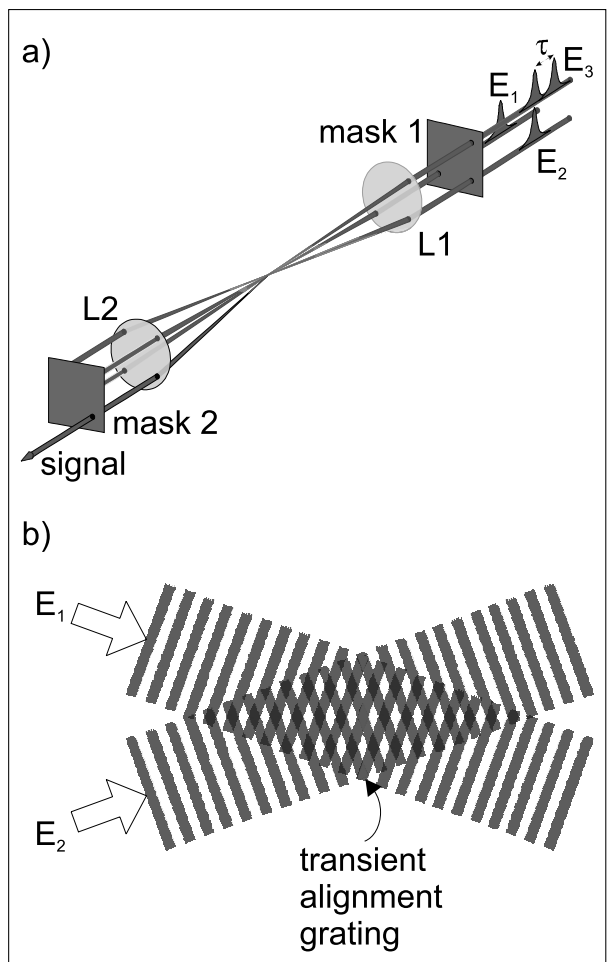

Fig. 3. a) Experimental arrangement for DFWM: The fs pulses $E_{1}$ (pump) and $E_{2}$ (dump) enter from top right through mask 1 and are focused into the sample by lens L1. The overlapping beams form an alignment grating in the gaseous sample via the pump-dump stimulated Raman process, $c f$. Fig. 2a). A small part of the time-delayed probe pulse $E_{3}$ is scattered from the transient alignment grating formed by $E_{1}$ and $E_{2}$, resulting in a signal pulse that is recollimated by lens $\mathrm{L} 2$ and passed through the second mask. b) Transient grating formed by the electric fields $E_{1}, E_{2}$. Areas of higher absolute electric fields are darker. ergy of the $n$th Raman transition and $\Gamma$ the rate of collisional dephasing in the sample.

\section{Experimental Setup}

Our experimental setup employed for recording fs-DFWM spectra has been described [9]: The output of a Maitai (Spectra Physics) Ti:sapphire oscillator is amplified at a repetition rate of $500 \mathrm{~Hz}$ with a Quatronix femtosecond chirped-pulse amplifier, giving pulses of $75 \mathrm{fs}$ FWHM centered at $800 \mathrm{~nm}$; the measured spectral bandwidth is $12.63 \mathrm{~nm}$ and the time-bandwidth product is 0.45 . The time and bandwidth properties of the fs pulses were characterized with a single-shot second-harmonic-generation frequency-resolved optical gating device (Grenouille, Swamp Optics).

The output is attenuated to $<60 \mu \mathrm{J}$ total and split into two pump beams and a probe beam of identical energy and polarization. The two pump beams are focused by a lens of focal length $f=1000 \mathrm{~mm}$ into a stainlesssteel gas cell containing the sample. The probe beam runs over a retroreflector mounted on a computer-controlled delay stage with a length of $650 \mathrm{~mm}$ with a resolution of 1 $\mu \mathrm{m}$. To increase the accuracy of the delay measurement, a glass ruler (Sony BL55NEA) with nearly zero thermal expansion coefficient and a resolution of $\leq 0.1 \mu \mathrm{m}$ was mounted on the delay stage. To avoid environmental influences the stage was operated under vacuum $\left(10^{-1} \mathrm{mbar}\right)$. Time zero was established by maximizing the Kerr signal of Ar gas in the cell (6.5 mbar). The total delay time of $1.3 \mathrm{~ns}$, which covers about 50 full recurrences of $\mathrm{C}_{3} \mathrm{H}_{6}$, was scanned in overlapping regions of $\approx 400 \mathrm{ps}$ length in steps of $33.3 \mathrm{fs}$. The DFWM signal beam is split and simultaneously detected by two separate photomultipliers. The high-intensity signals are recorded with a Hamamatsu R928 while the low-intensity wings of the transients are measured with a cooled RCA C31034 GaAs photomultiplier. The signals are digitized with a LeCroy WP954 transient digitizer, scaled, combined and stored in a PC. Cyclopropane (Aldrich) was purified by a several freeze-pump-thaw cycles to remove any residual contamination. Measurements were performed at 26.0 mbar. The pressure was measured with an MKS Baratron capacitance gauge accurate to \pm 0.1 mbar.

\section{Results}

Fig. 4 shows the fs-DFWM RCS transient of cyclopropane up to $1325 \mathrm{ps}$. The signal at time-zero is about six times as intense as the first full recurrence and is off the scale in Fig. 4. Two sets of rotational recurrences can be observed: The so-called 'half' recurrences with a spacing of $\approx 12.5 \mathrm{ps}$ are due to rotational Raman transitions with $\Delta \mathrm{J}= \pm 2$, the full recurrences with spacings of $\approx 25$ ps originate from the superimposed $\Delta \mathrm{J}= \pm 1$ and $\Delta \mathrm{J}= \pm 2$ Raman transitions. Due to collisional dephasing, the overall signal decays with an effective dephasing constant $\Gamma=7 \times 10^{-9} \mathrm{~s}^{-1}, c f$. Eqn. (6). The RCS transient is fitted with a microscopic time and rotational state dependent model [10].

The additional peaks towards longer times appear due to the positive centrifugal constant $D_{\mathrm{J}}$, those to shorter times are due to $\mathrm{D}_{\mathrm{JK}}$, which is negative in cyclopropane. The modulation intensity of these additional peaks increases with increasing size of the centrifugal distortion constants. The full recurrences are more complex than the half recurrences, due to the interference of the two transitions with $\Delta \mathrm{J}= \pm 1$ and $\Delta \mathrm{J}= \pm 2$ with different Clebsch-Gordan coefficients. The half recurrences consist of a series of equally spaced peaks with an alternation in intensity due to centrifugal effects and/or non resonant background.

The initial fits of rotational constants were performed for each individual recurrence shown in Fig. 4. The final fit was performed for the entire data set, encompassing $>100$ recurrences. The Table shows the resulting values: The rotational constant $\mathrm{B}_{0}$ $=20.093322 \mathrm{GHz}$ agrees with the value obtained by dipole distortion microwave spectroscopy [21] to within $0.012 \mathrm{MHz}$ and lies within the joined $1 \sigma$ standard deviations of the fit. The uncertainty of the microwave determination is about four times smaller that of our fs-DFWM measurement [21]. Note that our uncertainty for $\mathrm{D}_{\mathrm{JK}}$ is larger than that of $\mathrm{D}_{\mathrm{J}}$, since $\mathrm{D}_{\mathrm{JK}}$ depends not only on $\Delta \mathrm{J} \neq 0$ but also on $\Delta \mathrm{K} \neq 0$ transitions, which are not observed here due to the Raman selection rules.

\section{Conclusion}

The $\mathrm{B}_{0}, \mathrm{D}_{\mathrm{J}}$ and $\mathrm{D}_{\mathrm{JK}}$ constants of cyclopropane have been accurately measured using femtosecond degenerate four-wave mixing. Rotational coherence transients were recorded over a delay time of $1325 \mathrm{ps}$. The analysis is based on rotational Raman transitions of a symmetric nonrigid top. We determine $B_{0}$ with a relative accuracy of $\approx 7$ $\times 10^{-7}$ and $\mathrm{D}_{\mathrm{J}}, \mathrm{D}_{\mathrm{JK}}$ with relative accuracies of $\approx 10^{-3}$. To achieve this, we have implemented high-accuracy $(\leq 0.1 \mu \mathrm{m})$ measurements of the optical delay distance. Furthermore, we operate the delay stage under vacuum, to eliminate the uncertainties of the refractive index of the air due to varying temperature, pressure, humidity and $\mathrm{CO}_{2}$ concentration. The fs-DWFM $\mathrm{B}_{0}$ rotational constant deviates by only $0.012 \mathrm{MHz}$ from that previously measured by Brupbacher $e t$ $a l$. using dipole distortion microwave spectroscopy [21]. On the other hand, our value 


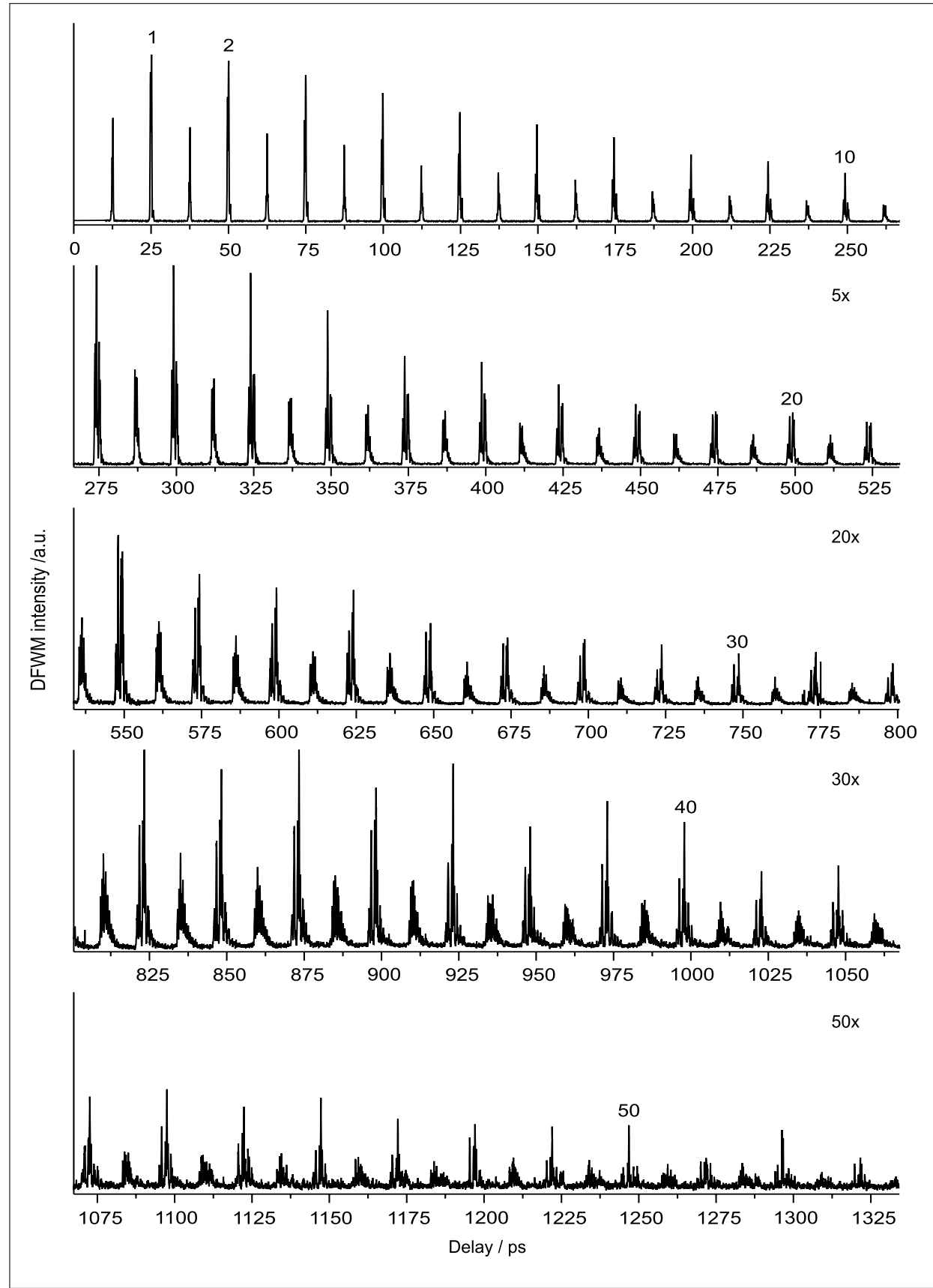

Fig. 4. Femtosecond degenerate four-wave mixing (fs-DFWM) rotational coherence

Table. Experimental rotational constants $(\mathrm{GHz})$ and centrifugal distortion constants $(\mathrm{kHz})$ of cyclopropane

\begin{tabular}{llll} 
Parameter & fs-DFWM & Fourier transform infrared & Fourier transform microwave \\
\hline$B_{0}$ & $20.093322(12)$ & $20.09332(3)$ & $20.0933348(28)$ \\
$C_{0}$ & $12.522(90)$ & $12.5557498(18)$ \\
$D_{J}$ & $29.346(55)$ & $28.985(29)$ & $28.9803(53)$ \\
$D_{\text {JK }}$ & $-36.09(13)$ & $-37.447(87)$ & $-37.4512(37)$
\end{tabular}

aThis work, brefs. [22][23], ' ref. [21] is more accurate than the $\mathrm{B}_{0}$ derived from high-resolution Fourier transform infrared spectroscopy [22][23]. This study shows the potential of the fs-DWFM method and serves as a benchmark for further investigations.

\section{Acknowledgements}

This work was supported by the Schweiz. Nationalfonds (project 200021-102030).

Received: February 19, 2006

[1] D.W. Pratt, Annu. Rev. Phys. Chem. 1998, $49,481$.

[2] A. Domenicano, I. Hargittai, 'Accurate Molecular Structures', Oxford University Press, Oxford, 1992.

[3] D. Papousek, M.R. Aliev, 'Molecular Vibrational-Rotational Spectra', Elsevier Scientific, New York, 1982.

[4] G. Herzberg in 'Molecular Spectra and Molecular Structure', Vol. II, Van Nostrand Reinhold, Malabar, 1945, p. 23.

[5] H. W. Kroto, 'Molecular Rotation Spectra', Dover Publications, Inc., 2003.

[6] P. Felker, A.H. Zewail, chapter 5 in 'Femtosecond Chemistry', Vol. 1, Eds. J. Manz, L. Wöste VCH, Weinheim, 1995, p. 193.

[7] P.M. Felker, J. Phys. Chem. 1992, 96, 7844.

[8] H.M. Frey, P. Beaud, T. Gerber, B. Mischler, P.P. Radi, A. P. Tzannis, Appl. Phys. B: Lasers Opt. 1999, 68, 735.

[9] H.M. Frey, A. Müller, S. Leutwyler, J. Raman Spectrosc. 2002, 33, 855.

[10] D.S. Kummli, H.M. Frey, M. Keller, S. Leutwyler, J. Chem. Phys. 2005, 123.

[11] J.S. Baskin, P.M. Felker, A.H. Zewail, J. Chem. Phys. 1987, 86, 2483.

[12] P.M. Felker, A.H. Zewail, J. Chem. Phys. 1987, 86, 2460.

[13] C. Riehn, Chem. Phys. 2002, 283, 297.

[14] T. Lang, M. Motzkus, H.M. Frey, P. Beaud, J. Chem. Phys. 2001, 115, 5418.

[15] C. Riehn, M.I. Kunitski, V.V. Matylitsky, M.F. Gelin, B. Brutschy, Phys. Chem. Chem. Phys. 2005, 7, 3955.

[16] C. Riehn, V.V. Matylitsky, M.F. Gelin, B. Brutschy, Mol. Phys. 2005, 103, 1615.

[17] W. Jarzeba, V.V. Matylitsky, C. Riehn, B. Brutschy, Chem. Phys. Lett. 2003, 368, 680.

[18] A.C. Eckbreth, Appl. Phys. Lett. 1978, 32, 421.

[19] J.A. Shirley, R.J. Hall, A.C. Eckbreth, Opt. Lett. 1980, 5, 380.

[20] E.J. Brown, Q.G. Zhang, M. Dantus, J. Chem. Phys. 1999, 110, 5772.

[21] T. Brupbacher, C. Styger, B. Vogelsanger, I. Ozier, A. Bauder, J. Mol. Spectrosc. 1989, 138, 197.

[22] J. Pliva, J.W.C. Johns, J. Mol. Spectrosc. 1985, 113, 175.

[23] J. Pliva, J.W.C. Johns, Can. J. Phys. 1984, $62,1369$. 